\title{
Use of Concept Generation Techniques in Different Cultural Settings
}

\section{Ms. Meagan R. Vaughan, University of Texas, Austin}

Meagan R. Vaughan received a B.S. in Engineering, Mechanical Engineering concentration, in 2007 from LeTourneau University in Longview, TX, USA. During her time at LeTourneau University, she was an active participant for two years in the LeTourneau Engineering Global Solutions (LEGS) program, now LIMBS International, designing low-cost lower limb prosthetic components for developing countries. She received a M.S. in Engineering with a concentration in Biomechanics in 2009 and is currently pursuing a Ph.D. at The University of Texas at Austin, Austin, TX, USA.

\section{Dr. Richard H. Crawford, University of Texas, Austin}

Dr. Richard H. Crawford is a Professor of Mechanical Engineering at The University of Texas at Austin and is the Temple Foundation Endowed Faculty Fellow No. 3. He is also Director of the Design Projects program in Mechanical Engineering. He received his BSME from Louisiana State University in 1982, and his MSME in 1985 and Ph.D. in 1989, both from Purdue University. He teaches mechanical engineering design and geometry modeling for design. Dr. Crawford's research interests span topics in computeraided mechanical design and design theory and methodology. Dr. Crawford is co-founder of the DTEACh program, a "Design Technology" program for K-12, and is active on the faculty of the UTeachEngineering program that seeks to educate teachers of high school engineering. 


\title{
Use of Concept Generation Techniques in Different Cultural Settings
}

\begin{abstract}
Systematic design processes that utilize concept generation tools, such as 6-3-5/C-Sketch and Brainstorming with Mind-Mapping, are widely applied in product development processes in developed countries. The use of these tools contributes to improvements of many common devices. Designers from developed countries also apply these concept generation tools to solve design problems in developing countries. In order to successfully design products for developing countries, leaders in low-cost product design advocate the inclusion of the end-user as a means to more accurately identify and satisfy the needs of people in these countries. However, efforts by engineers to include the end-user, and ultimately train them to lead future design efforts, often adds an additional layer of complexity due to differences in culture and in language among participants.
\end{abstract}

To transfer these techniques to designers in developing communities, it is important to first determine the extent to which these tools are culture-specific, what modifications must be made in order for them to be effective in other cultures, and how these might affect the teaching of the techniques. This paper presents a pilot study in which designers in a developing country were taught to use concept generation techniques. It is hypothesized that methods to make these concept generation techniques effective in other cultures will become apparent from participant feedback and results.

Since the ability of participants to learn and communicate ideas is critical for the success of concept generation, two concept generation techniques were selected for their ability to accommodate multiple learning styles - visual, auditory, and kinesthetic. The selected techniques, Brainstorming with Mind Mapping and 6-3-5/C-Sketch, are kinesthetic in nature and rely on active participation, writing, and/or sketching by all group members. They are differentiated by the secondary learning style they encourage the participant to utilize.

Study participants were recruited from a prosthetic clinic in Bolivia. They were taught how to apply these concept generation methods to a shared problem - to create a prosthetic socket design that accommodates changes in residual limb volume. This problem was chosen because lack of proper fit and comfort in their sockets continues to be an issue of great concern to amputees, despite improved technology and advancements in understanding lower limb prosthetic socket fit. Existing prosthetic socket technology often cannot compensate for changes in the shape of the residual limb and the resulting discomfort for the user. Therefore, a new socket design is sought using these techniques.

Based on the results of the study, the more visual techniques, such as the 6-3-5/C-Sketch method, were more widely accepted while the verbal techniques were not. This outcome suggests that removing the language barrier may encourage effective concept generation when crossing cultural boundaries. 


\section{Introduction}

Systematic design processes that utilize concept generation tools, such as 6-3-5/C-Sketch and Brainstorming with Mind-Mapping, are widely applied in product development processes in developed, industrialized countries. The use of these tools contributes to improvements to many common devices, from nail clippers to handheld power screwdrivers. ${ }^{1}$ Designers from developed countries also apply these concept generation tools to solve design problems in developing countries. There are many examples of engineers, including students and professionals from organizations like Engineers Without Borders (http://www.ewb-usa.org), who apply their design expertise to improve quality of life through high quality, low-cost product designs. ${ }^{2-3}$ While the intentions are always good, occasionally these products are left unusable due to minor complications - poor maintenance, a standard part is lost, etc. due to lack of knowledge or skill in the receiving community. In some cases, the design may simply miss the real needs of the receiving community.

To design a more successful product, active inclusion of the end-user in the design process can help engineers from developed countries more fully understand the needs (both spoken and unspoken) of users in developing countries. Leaders in low-cost product design advocate this inclusion of the end-user as a means to more accurately identify and satisfy the needs of people in these countries. ${ }^{4-5}$ However, efforts by engineers to include the end-user, and ultimately train them to lead future design efforts, often add an additional layer of complexity due to differences in culture and in language among participants.

To transfer these techniques to designers in developing communities, it is important to first identify the cultural assumptions and biases inherent in the methods. Since the cultural context can also inform the teaching of the techniques, this knowledge can be used to modify the methods to make them effective in other cultures. This paper presents a pilot study in which designers in a developing country were taught to use concept generation techniques. It is hypothesized that methods to make these concept generation techniques effective in other cultures will become apparent from participant feedback and results. After reviewing the results of this concept generation session, preliminary recommendations for changes to accepted concept generation techniques are presented for overcoming cultural barriers to the inclusion of users from multiple cultures in the design process.

\section{Background}

\section{Concept Generation Techniques}

As the ability of participants to learn and communicate ideas is critical for the success of concept generation, two concept generation techniques were selected based on their ability to accommodate multiple learning styles - Visual, Auditory, and Kinesthetic. Both techniques, Brainstorming with Mind Mapping and 6-3-5/C-Sketch, are kinesthetic in nature and rely on active participation, writing, and/or sketching by all group members. They are differentiated by the secondary learning styles they encourage the participants to utilize. 
Brainstorming with Mind Mapping relies on the verbal communication of ideas during a group ideation session. ${ }^{1}$ As participants generate ideas, they map them out in a web based on their interconnection to the central design problem (Figure 1, left). This mapping structure helps focus the group on areas where additional innovation is needed.

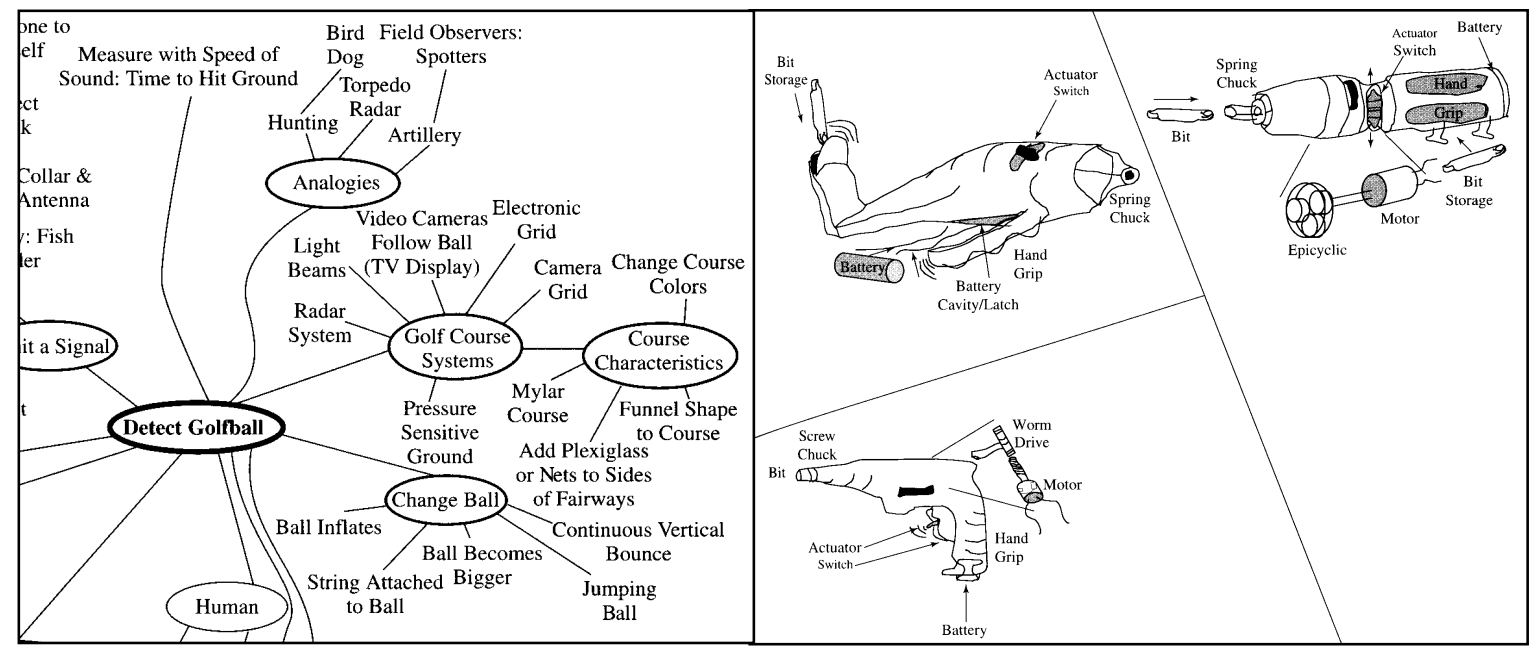

Figure 1 - Examples of Concept Generation Methods: Left - Portion of a Mind Map for "Detect a Golf ball" and Right - The 6-3-5 Method applied to a power screwdriver product ${ }^{1}$

In comparison, C-Sketch (based on the 6-3-5 method) relies on visual communication of ideas based on communal sketching. During C-Sketch, a group of 6 people are given sheets of paper on which to draw 3 initial ideas that address the central design problem (Figure 1, right). Participants are given time to sketch their ideas and then pass their sheets of paper on to the next person in their group, completing a round of drawing. With their neighbor's drawings in front of them, participants add to the drawings for a period of time before passing it along, completing another round. This is repeated until each participant has had an opportunity to add to the sketches of all other participants and their sheet is returned to them $(\sim \mathbf{5}$ rounds total $) .{ }^{1}$ This technique also helps to ensure that one or two individuals do not dominate the ideation process by requiring all participants to remain silent during all rounds of sketching.

\section{Design Problem Description}

Due to ongoing issues with the quality of prosthetic components in developing countries, a socket design that accommodates changes in residual limb volume was selected as the central design problem for this case study. In spite of advancements made in understanding lower limb prosthetic socket fit and improvements in socket technology, the lack of proper fit and comfort in an amputee's socket continues to be an issue of great concern. ${ }^{6-9}$ Although "currently available components offer improved function and superior symmetry of gait...The focus on the features of these components sometimes has led, however, to neglect of the basic elements of prosthetic design-the fit and the alignment."

This lack is often a result of changes in the shape of the residual limb that existing technology cannot accommodate. This shape change, causing either an increase or decrease in volume of the residual limb, is produced by a variety of factors, such as weight gain/loss, 
atrophy, swelling or normal adolescent growth. ${ }^{10-13}$ A socket design that accommodates these changes in residual limb volume is crucial. In regions worldwide where cost is a prohibitive factor in the replacement of ill-fitting prosthetic sockets, the need for a volume adjustable, and therefore longer lasting, socket is widespread.

\section{Methods}

The sequence of procedures used in this study is based on a typical product design process outlined by Otto and Wood. ${ }^{1}$ Following participant recruitment, the study procedures include the six steps summarized in Figure 2 and described below.

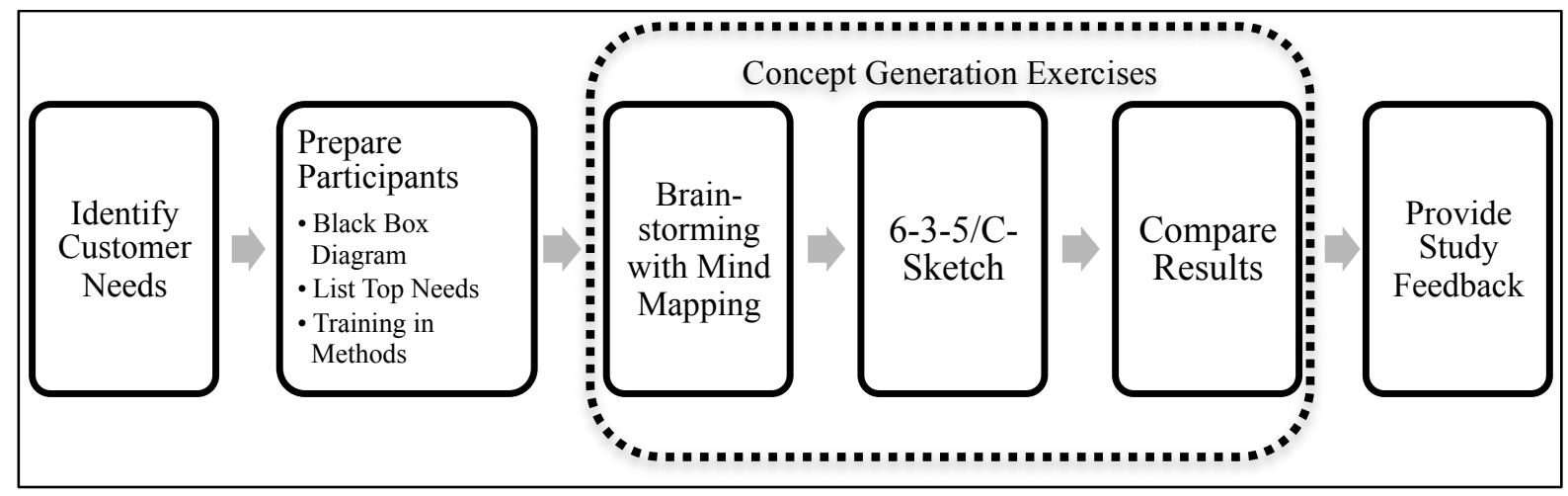

Figure 2 - Summary of study procedures

\section{Participant Population}

Participants were recruited through their established relationships to Centro de Miembros Artificiales, a prosthetic clinic located in La Paz, Bolivia (www.rotaryboliviaprosthetics.org). During the time of recruitment, participants were identified based on their presence in the clinic waiting area. Participants were informed of the nature of the study before beginning.

Participants were assigned to one or both of the study sub-groups, based on their personal preference, into the Customer Needs Analysis (CNA) Group and/or Concept Generation (CG) Group. Regardless of the assigned group, criteria for inclusion in the study were based on having, or caring for someone who has, a lower limb amputation. Adults (18-80yrs) of all genders, ethnicities, and health levels were included in the subject population. Children ( $<18 y r s)$ or individuals with significant cognitive issues or visual impairment were excluded.

The investigator that facilitated, but did not participate in, the concept generation spoke English only and had limited previous experience with teaching through an interpreter. Therefore a translator was present to assist those who spoke Spanish only or English only. Participants were encouraged to contribute ideas in their native language, Spanish. All study data was translated into English for analysis.

The target sample size for this pilot study was based on the number of participants required for the Concept Generation exercises and the number of interviews necessary to determine customer needs. As described by Otto and Wood, 6-3-5/C-Sketch is traditionally completed in groups of six participants. ${ }^{1}$ Therefore, a minimum of six concept generation 
participants was required in the CG Group. In determining the required number of participants for the CNA Group, it has been demonstrated that 20-30 interviews are necessary to obtain 90$95 \%$ of the customer needs for a given design problem. ${ }^{14}$

\section{Identify Customer Needs}

Customer Needs, those aspects, functions, and features users identify as necessary in a product they use, were first determined by observation of the clinic staff and patients in the CNA Group. During observation, a study facilitator recorded behavioral patterns and uses of prosthetic devices by participants. In addition, individual interviews were completed based on a predetermined set of interview questions about socket comfort and usage. As needed, an interpreter was on hand to help with translation and audio was recorded for transcription purposes.

\section{Participant Preparation and Training}

At a separate CG Group meeting, a series of preliminary exercises was used to help participants think about all facets and requirements of the design problem chosen for this study. Training in the concept generation techniques of interest in this study was also provided.

Participants were instructed to use a Black Box Diagram ${ }^{\dagger}$ for the function statement: Change Socket Volume. Beginning concept generation using this diagram was intended to help the participant group to identify all inputs and outputs for the system. ${ }^{1}$ In addition, this allowed the participants to begin discussing the possible customer needs that were important for them to address in the design.

Participants were then instructed to generate a list of the top needs, or requirements, an amputee in their community would have for an appropriate socket design. This list was compared and combined with results from the Customer Needs Analysis (described above) and used in future steps.

With this list of customer needs, the group received instruction, in a lecture style setting, on the Brainstorming with Mind Mapping and 6-3-5/C-Sketch concept generation methods. Again, during these exercises, the investigator facilitated and a translator was utilized as needed.

\section{Concept Generation Exercises}

After completion of the training and warm-up exercises, the CG group was guided through the concept generation exercises. For these exercises, the investigator provided the necessary materials (paper and colored writing implements) and seated participants around a table. Each participant was provided with a different color pen to use throughout each exercise and to keep after participating in the study. The different colored pens enable participants and investigators to identify individual contributions. However, the investigator did not maintain records connecting individuals to the color pen used.

\footnotetext{
${ }^{\dagger}$ A Black Box Diagram is a graphical method for identifying the flow of energy, material, and signals through a hypothetical product, i.e. the Black Box. ${ }^{1}$
} 
During the Brainstorming with Mind Mapping exercise, participants were encouraged to write in their native language, while mapping out ideas for the seed phrase, Change Volume, on a large sheet of paper. Participants were encouraged to take as much time as needed to exhaust all areas of their mind map ( $45 \mathrm{~min})$.

Using the top customer needs and ideas previously generated, participants were next instructed to sketch possible designs for a volume adjustable socket using the 6-3-5/C-Sketch method. Participants were instructed to limit written and spoken words during this exercise and to focus on communicating ideas graphically. As this exercise is completed in rounds, participants were instructed to pass the drawings they were working on to the next person only when all participants declared they were finished or the time limit was reached. The time limits per round were 5-15 min for the first round and 2-5 min for each subsequent round.

During these exercises, the investigator recorded observations about the group dynamic and their execution of the exercises. A one-hour lunch break was provided between exercises. After completion of all concept generation exercises, participants were encouraged to review all concepts and discuss any modifications.

\section{Feedback}

Participants were given the opportunity to give feedback to the investigator on the concept generation exercises and the generated concepts. A nine-item questionnaire was provided for participants to complete (Table 1). The investigator remained on hand to answer any questions that arose but encouraged participants to complete the surveys independently.

Table 1 - Post Concept Generation Survey Questions

1. In your opinion, what is the most useful method for coming up with ideas/concepts (feel free to list methods not used today):

2. Of those methods used today, which did you like/prefer? Why?

3. Did you dislike either or both of the methods? Why?

4. Did you have trouble understanding how either of the methods worked?

5. Are you a visual, auditory, kinesthetic, etc. learner?

6. How would you change any of these methods?

7. Do you feel like the group was able to come up with good results? Why/why not?

8. Which idea was your favorite?

9. How old are you?

\section{Results}

Data was collected at Centro de Miembros Artificiales using the methods described above. During processing, the data was translated into English for analysis. To ensure that the full intent of the writer was communicated through the translation, a participant in the study (primary language Spanish), rather than a third party, completed the translation of all concept generation results from Spanish to English. A second translator, who also participated in the concept generation processes, verified this translation (primary language English). 


\section{Participant Demographics}

Demographics were recorded for the recruited participants (Table 2). Of the participants, some spoke Spanish-only while others were bilingual, Spanish-English. Participants were divided into study sub-groups, Customer Needs Analysis (CNA) Group and/or Concept Generation (CG) Group, based on their desired level of participation.

Table 2 - Participant Sub-Group Demographics

\begin{tabular}{lcccc}
\hline Study Group & Male & Age(Stdev) & Female & Age(Stdev) \\
\hline CNA Group & 12 & $49(17)$ & 3 & $45(15)^{*}$ \\
CG & 6 & $54(8)$ & 1 & 60 \\
Both CNA and CG Groups & 4 & $51(5.5)$ & 0 & \\
\hline Total (CNA+CG-Both) & $\mathbf{1 4}$ & $\mathbf{5 0}(\mathbf{1 6})$ & $\mathbf{4}$ & $\mathbf{5 0 ( 1 4 )}$ \\
\hline${ }^{*}$ Only two reported age. & & & &
\end{tabular}

This subject population represented a range of socioeconomic and education levels. The population included employed and unemployed, educated and uneducated participants. There were educated medical doctors, tradesmen who worked as technicians at the clinic, and unskilled shopkeepers.

\section{Black Box Diagram}

The Concept Generation (CG) group successfully created a Black Box Diagram to map sources of energy, material, and information pertinent to the device for the function statement: Change Socket Volume (Figure 3, top).

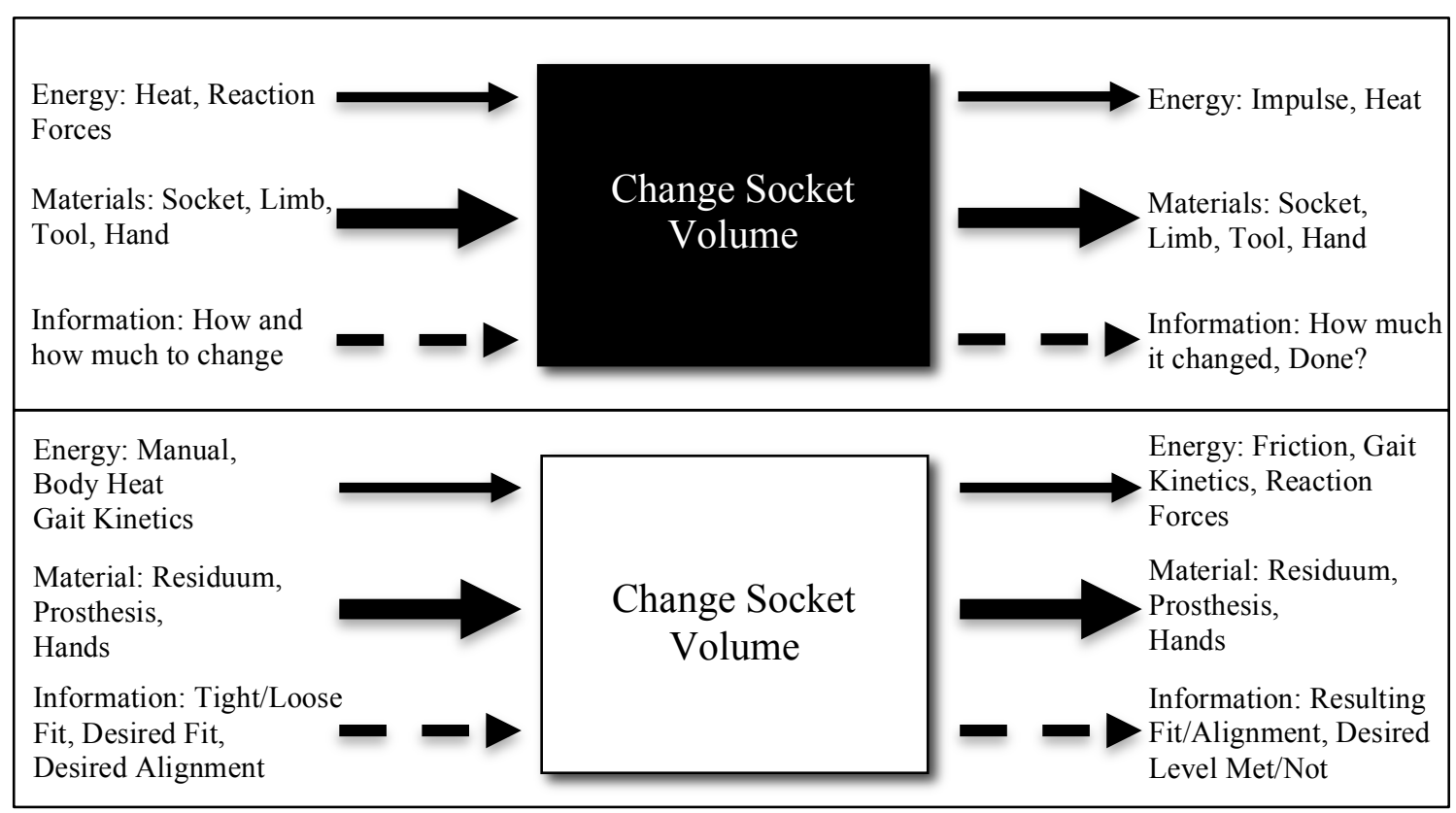

Figure 3 - Bolivian (Top, Translated from Spanish) and Authors' (Bottom) Black Box Diagram mapping flows of energy, material, and information through a potential product represented as a function statement 
For comparison, a Black Box Diagram generated by the authors in advance (Figure 3, bottom) shows the sources of energy, material, and information deemed important by the authors based on personal experience and a literature review. The Bolivian participants produced a useful Black Box Diagram that differed from the authors' diagram primarily in word choice, e.g. reaction forces vs. gait kinematics. Their Black Box Diagram lacked depth in the more abstract areas of energy and information; they did not include manual and friction energy or address alignment.

\section{Top Needs}

The CG group brainstormed a list of needs for an appropriate socket design for amputees in a developing community. The Bolivian participants identified 10 customer needs during this session (Table 3). For comparison, the responses from the interviews with participants in the CNA group were restated as interpreted needs by the authors using the techniques described by Otto \& Wood ${ }^{1}$ and Becker \& Patnaik. ${ }^{15}$

Table 3 - Comparison of Customer Needs generated by participants during the Concept Generation session and developed by the authors from responses in the Customer Needs Analysis interviews (Shaded needs were identified, at some level, by both groups)

\begin{tabular}{lll}
\hline Customer Needs Based on & & Customer Needs Based on \\
CG Group Brainstorming & & Responses from CNA Group \\
\hline - Low Cost & - Low Cost \\
- Volume Adjustable & - Accommodate Volume Change \\
- Simple Maintenance & - Easy to Maintain by user \\
- Long Life & - Long Life \\
- Simple to Adjust & - Simple to Adjust by users \\
- Pretty & - Socially Acceptable \\
- Local Materials & - Simple/Local Manufacturing \\
- Light & - Maintain Proper Biomechanics \\
- Comfortable & - Work in wet/dusty environment \\
- Safe & \\
\hline
\end{tabular}

As shown in Table 3, the CG group successfully identified $89 \%$ of the customer needs identified from the CNA interview response. Of these, they specifically identified $56 \%$ and partially identified $30 \%$ of the needs.

\section{Brainstorming with Mind Mapping}

As instructed, the group completed the Brainstorming with Mind Mapping exercise for the seed phrase: Change Volume (Figure 4). Individual contributions were identified through the use of different color pens (black was used by the investigator for translation purposes and to draw in the connections of the mind map). 


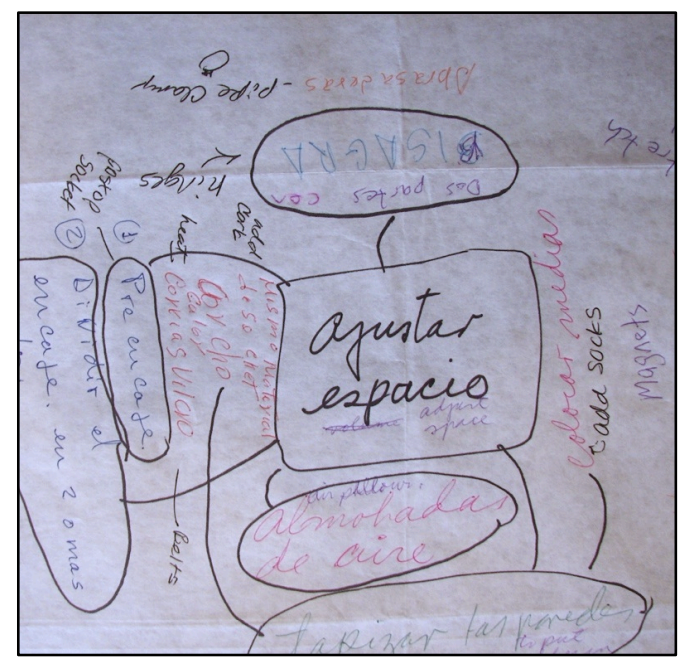

Figure 4 - Section of the Mind Map from participants (including English translations)

Twenty-two concepts were recorded during the 45-minute group brainstorming session with each participant contributing, on average, 3.14 (SD 2.67) concepts (Table 4). The high standard deviation in average contributions-per-participant is due to the 'Navy' participant's contribution of $40 \%$ of the concepts. On average, each individual contributed at a rate of 0.07 contributions/min (or 1 concept every $15 \mathrm{~min}$ ) and, as a result, the group contributed concepts at a rate of 0.49 concepts/min (or 1 concept every 2 minutes). Participants identified no subcategories during the exercise.

Table 4 - Results of Brainstorming with Mind Mapping Exercise

\begin{tabular}{|c|c|c|c|c|c|}
\hline Participant & & & & Total & $\%$ Of Total \\
\hline Pink & Add Socks & Pieces of Cotton & Air Pillow & 3 & $13.64 \%$ \\
\hline Orange & Suction Valves & Pipe Ring/Clamp & & 2 & $9.09 \%$ \\
\hline Red & Add Cork & Velcro Belt & Heat & 3 & $13.64 \%$ \\
\hline \multirow[t]{3}{*}{ Navy } & Silicon & Expandable & Post-op Socket & & \\
\hline & Divide into $2^{+}$parts & Diet & Explore Anatomic Shapes & & \\
\hline & Depends on Diagnosis & Anti-inflammatory & Osseointegration & 9 & $40.91 \%$ \\
\hline Green & Put rubber in walls & Plastic Foam & & 2 & $9.09 \%$ \\
\hline Light Blue & Two Parts w/Hinges & & & 1 & $4.55 \%$ \\
\hline \multirow[t]{2}{*}{ Purple } & Magnets & Stretch & & 2 & $9.09 \%$ \\
\hline & & & & 22 & $100 \%$ \\
\hline
\end{tabular}

\section{6-3-5/C-Sketch}

Participants completed the 6-3-5/C-Sketch exercise to generate possible designs for the volume adjustable socket. Samples of sketches generated are show in Figure 5 below. These sketches illustrate the proposed use of hinged sections, varying shapes of inserts, inflatable inserts, and pipe clamps to regulate available space within the socket. 


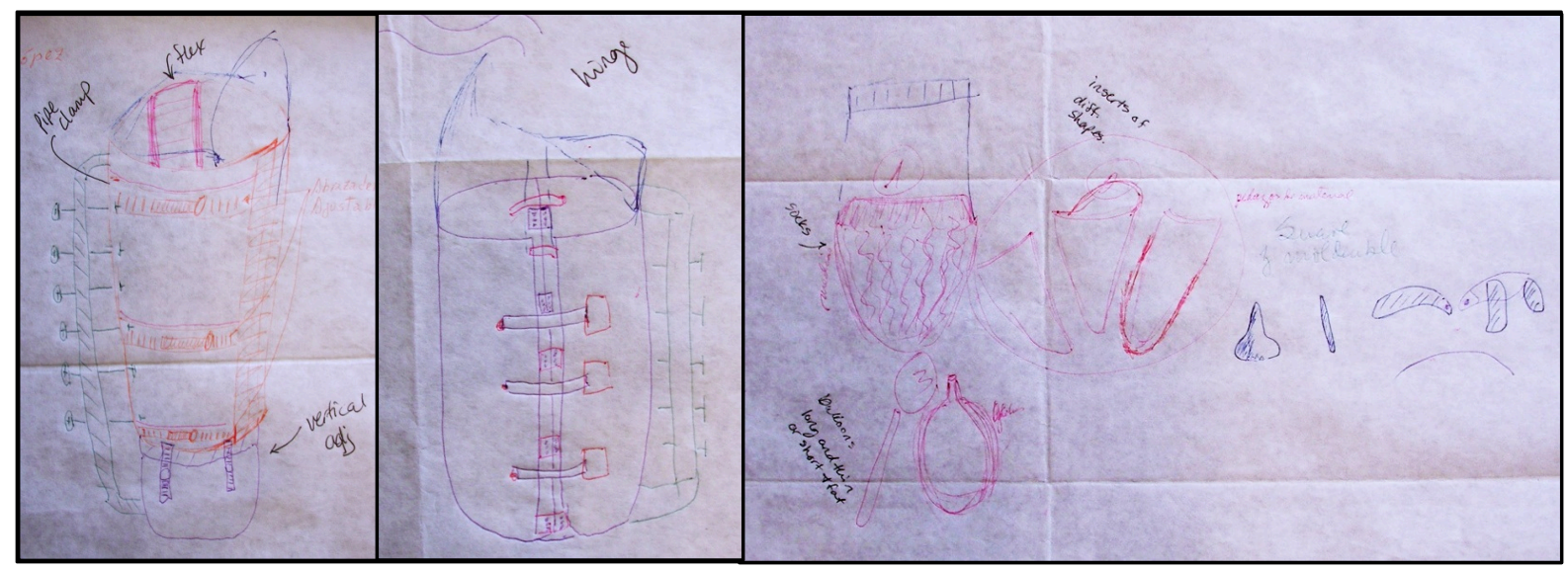

Figure 5 - Sample sketches from Bolivian 6-3-5/C-Sketch

\section{Feedback}

The feedback from the nine-item questionnaire showed the 6-3-5/C-Sketch Method was preferred by 5 of the 7 participants (Table 5). Of these five participants, all were self-reported kinesthetic and/or visual learners.

Table 5 - Preferred Concept Generation techniques and learning styles reported by participants

\begin{tabular}{|c|c|c|c|c|c|c|c|}
\hline \multirow{5}{*}{ 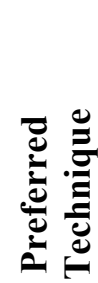 } & \multicolumn{7}{|c|}{ Preferred Learning Style } \\
\hline & & Visual & $\begin{array}{c}\text { Vis. \& } \\
\text { Aud. }\end{array}$ & Kinesthetic & $\begin{array}{c}\text { Kin. \& } \\
\text { Vis. }\end{array}$ & Depends & Total \\
\hline & 6-3-5/C-Sketch & 1 & 0 & 2 & 1 & 1 & 5 \\
\hline & Mind Map & 0 & 0 & 0 & 1 & 0 & 1 \\
\hline & Black Box & 0 & 1 & 0 & 0 & 0 & 1 \\
\hline & Total & 1 & 1 & 2 & 2 & 1 & 7 \\
\hline
\end{tabular}

The two participants that did not prefer 6-3-5/C-Sketch preferred the Black Box Diagram and the Mind Mapping exercise respectively. The reported preferred learning style of the participant that preferred the Black Box Diagram was visual and auditory while the Mind Mapping was kinesthetic and visual. Note that the participant that preferred the Mind Mapping exercise was the only non-Bolivian native in the group. Six of the participants, however, felt that they were able to obtain good results from these exercises. None of the participants reported difficulty in understanding how to execute the concept generation exercise.

Of the three participants that expressed a dislike for one of the exercises, they tended to dislike the technique that was in contrast to their preferred, i.e. if they preferred C-Sketch they tended to dislike the Mind Mapping exercise. Specific feedback from one participant about the Black Box Diagram was that they "did not like it because it was too abstract". Another disliked the Mind Mapping exercise due to the fact that "it encouraged people to write unreal ideas".

\section{Discussion}

A product design process was conducted with participants from a developing community in an effort to determine the extent commonly implemented concept generation tools are culture 
specific and to identify modifications that must be made for them to be effective in other cultures. Based on the results of the feedback surveys, the concept generation techniques were successfully taught and implemented in the Bolivian setting. The participants reported that they did not have trouble understanding the techniques and the group was able to generate good results. Of the techniques taught, the more visual 6-3-5/C-Sketch method was preferred over the more verbal Brainstorming with Mind Mapping exercise.

\section{Top Customer Needs}

Based on the comparison of needs generated by the Concept Generation (CG) group with those restated by the investigator from the Customer Needs Analysis (CNA) interviews, the CG group had a firm understanding of the design problem they were addressing. The participants identified approximately $90 \%$ of the needs generated by the CNA group. This was likely aided by the fact that four of the participants were part of both the CNA and CG groups.

\section{Learning Styles and Concept Generation Method}

When comparing learning styles with preferred concept generation methods, the proponents for the more visual sketching method also reported a preference for visual learning styles. In addition, the only participant that preferred the more auditory based Mind-Mapping exercise reported that their preferred learning style was also auditory. As anticipated, this suggests that participants tend to prefer concept generation techniques that closely mirror their own learning styles.

None of the participants reported difficulty in understanding how the concept generation techniques worked but some did have suggestions for improving the concept generation session. These suggestions included bringing a mock residual limb to the concept generation session, ensuring translation is completed ahead of time (rather than real-time translation), and focusing on the subject more.

\section{Concept Generation Session Observations}

It was observed that the Bolivian participants did not fully use the Mind Mapping strategy while brainstorming. They, instead, just listed concepts. Due to the investigator being unfamiliar with the local language, it was difficult to identify and dissuade this pattern during the exercise. As a result, participants may not have fully grasped the usefulness of the Mind Map. Therefore, this trend needs to be addressed in future implementations of this technique in this and other cultures.

In addition, a potential for a single participant to dominate the Brainstorming with Mind Mapping exercise was observed. Though instructed to participate equally and record their own ideas with their own colored pen, uneven participant distribution could be indicative of a single individual taking on a dominant role in the Brainstorming with Mind Mapping exercise. This was observed in the Navy participant contribution of $40 \%$ of the total concepts, nearly three times the entries on the mind map of the second highest contributors (Table 4). No treatment of this individual as a scribe was observed. Further, there is potential, due to the mixing of 
socioeconomic and education levels in this population, for there to be intimidation that could have further produced this dominant participant. Therefore, techniques for preventing an individual from dominating the exercise should be implemented in the future. This is not uncommon in group brainstorming sessions and techniques for minimizing this could include the enforcement of a circular sharing pattern or a variant of the Nominal Group Technique rather than a free-for-all. ${ }^{16}$

\section{Study Limitations}

As a pilot study, there are several limitations to this research. The primary limitation is sample size. In future research, additional concept generation groups in both Bolivia and other cultures would be beneficial. These additional groups would support additional statistical analysis while providing a basis for comparison between cultures. In order to compare concepts, quantification of Novelty, Quality, Variety, and Quantity has been proposed as a means to compare concepts generated by different groups. ${ }^{17-18}$

Another avenue for further study is the influence of age on participant contributions. One participant noted the relatively high age range of participants - the average age of CG participants was $54.6 \pm 7.5$ years. The introduction of younger participants could influence the dynamics of the group. As mentioned above, the impact of socioeconomic and education levels on the dominance of a single individual in generating concepts should also be addressed.

Additionally, one of the key complaints that participants had about the concept generation exercises was the added difficulty of learning the exercises through real-time translation of English to Spanish. It was suggested that everything be translated to Spanish in advance. This, coupled with the participants' preference for non-verbal concept generation techniques, suggests that removing the language barrier may encourage effective concept generation when crossing cultural boundaries.

\section{Conclusions}

Based on these preliminary results, the concept generation techniques studied were successfully taught and implemented in the Bolivian setting - all participants felt that they did not have trouble understanding the techniques and that the group was able to come up with good results. The more visual techniques, like 6-3-5/C-Sketch, were more widely accepted while the verbal techniques were not. Removing the language barrier may therefore encourage effective concept generation when crossing cultural boundaries but care must be taken to prevent dominant participants. This suggests that, though culture and language may play a role in changing the group dynamic, it does not prevent the use of these concept generation techniques in another culture.

\section{References}

1. Otto, K., \& Wood, K. (2000). Product Design: Techniques in reverse engineering and new product development. Upper Saddle River, NJ: Prentice Hall. 
2. Engineers Without Borders USA. Retrieved January 31, 2012, from http://www.ewb-usa.org/.

3. Gonzalez, R. V., \& Rispin, K. L. (2007). International Engineering Education - A Case Study of Working in Low-income Countries. Invited Lecture. ABET Annual Meeting - The Global Workforce: The future of Technological Education. Incline Village, NV.

4. Pollak, P. (2008). Out of poverty: What works when traditional approaches fail. San Francisco: BerrettKoehler Publishers, Inc.

5. Prahalad, C. K. (2010). The Fortune at the Bottom of the Pyramid: Eradicating Poverty Through Profits (5th anniversary ed., rev. and updated ed.). Upper Saddle River: Wharton School Publishing.

6. Dillingham, T. R., Pezzin, L. E., MacKenzie, E. J., \& Burgess, A. R. (2001). Use and Satisfaction with Prosthetic Devices Among Persons with Trama-Related Amputations. American Journal of Physical Medicine \& Rehabilitation 80, 563-571.

7. Legro, M. W., Reiber, G., Aguila, M. d., Ajax, M. J., Boone, D. A., Larsen, J. A., . . Sangeorzan, B. (1999). Issues of importance reported by persons with lower limb amputations and prostheses. Journal of Rehabilitation Research and Development, 36(3).

8. Pezzin, L. (2004). Use and satisfaction with prosthetic limb devices and related services. Archives of physical medicine and rehabilitation, 85(5), 723-729. doi: 10.1016/j.apmr.2003.06.002

9. Trower, T. A. (2006). Changes in Lower Extremity Prosthetic Practice. Physical medicine and rehabilitation clinics of North America, 17(1), 23-30.

10. Golbranson, F. L., Wirta, R. W., Kuncir, E. J., Lieber, R. L., \& Oishi, C. (1988). Volume changes occurring in postoperative below-knee residual limbs. Journal of Rehabilitation Research and Development, 25(2), 11-18.

11. Kegel, B., Burgess, E. M., Starr, T. W., \& Daly, W. K. (1981). Effects of Isometric Muscle Training on Residual Limb Volume, Strength, and Gait of Below-Knee Amputees. Physical Therapy, 61(10), 14191426.

12. Kurdibaylo, S. F. (1996). Obesity and metabolic disorers in adults with lower limb amputation. Journal of Rehabilitation Research and Development, 33(4), 387-394.

13. Smith, S. L., \& Buschang, P. H. (2005). Longitudinal models of long bone growth during adolescence. [Comparative Study]. American journal of human biology: the official journal of the Human Biology Council, 17(6), 731-745. doi: 10.1002/ajhb.20441

14. Griffin, A. and Hauser, J. R. (1993). The Voice of the Customer. Marketing Science, 12(1), pp.1-27.

15. Patnaik, D., \& Becker, R. (1999). Needfinding: the why and how of uncovering people's needs. Design Management Journal (Former Series), 10(2), 37-43.

16. Bartunek, J. M., \& Murninghan, J. K. (1984). The nominal group technique: expanding the basic procedure and underlying assumptions. Group \& Organization Management, 9(3), 417-432.

17. Shah, J. J., Smith, S. M., and Vargas-Hernandez, N., (2003). Metrics for Measuring Ideation Effectiveness. Design Studies, 24(2), 111-134.

18. Linsey, J. (2007). Design-by-Analogy and Representation in Innovative Engineering Concept Generation. $\mathrm{PhD}$, The University of Texas at Austin, Austin. 